\title{
Projection ablation lithography cathode for high-current, relativistic magnetron
}

\author{
M. C. Jones, V. B. Neculaes, R. M. Gilgenbach, ${ }^{\text {a) }}$ W. M. White, \\ M. R. Lopez, and Y. Y. Lau \\ Nuclear Engineering and Radiological Sciences Department, Intense Energy Beam Interaction Lab, \\ University of Michigan, Ann Arbor, Michigan 48109-2104
}

\section{T. A. Spencer}

Air Force Research Laboratory, Phillips Research Lab, Kirtland AFB, New Mexico

D. Price

Titan Corporation

(Received 5 March 2004; accepted 29 May 2004; published 14 September 2004)

\begin{abstract}
Initial results are presented of an innovative cathode operating in a relativistic magnetron powered by an accelerator with parameters: $-0.3 \mathrm{MV}, 1-10 \mathrm{kA}$, and $0.5 \mu$ s pulse length. This cathode is fabricated by ablating a pattern on the cathode using a $\mathrm{KrF}$ laser. This projection ablation lithography (PAL) cathode has demonstrated fast current turn-on and microwave startup times have decreased from an average of 193 to $118 \mathrm{~ns}$. The pulselength of $1 \mathrm{GHz}$ microwave oscillation has increased from a $144 \mathrm{~ns}$ average to $217 \mathrm{~ns}$. With these improvements in microwave startup and pulse length, the microwave power has approximately remained the same compared to the previously used cloth cathodes. A new triple-azimuthal emission region is tested as means of prebunching the electrons ("cathode priming") into the three spokes desired for pi mode operation in a six-cavity magnetron. The Tri-PAL cathode priming results in the fastest startup and highest efficiency of relativistic magnetron microwave generation. (C) 2004 American Institute of Physics.

[DOI: $10.1063 / 1.1784561]$
\end{abstract}

\section{INTRODUCTION}

There currently exist two types of microwave tube technology:

(a) commercial, high-vacuum, long-life thermioniccathode, repetitively-pulsed, or cw tubes, and

(b) experimental, high-power-microwave (HPM) tubes which operate single pulse, with poorer vacuum (typically $\sim 10^{-6}$ Torr) and expendable (cloth/fabric) cathodes.

The device presented in this article strictly falls into the second category. However, experimental results reported here use a ceramic insulator (base vacuum on the $10^{-8}$ Torr scale) and all-metal cathode (no cloth), which represent the first steps in a research program at The University of Michigan (UM) to apply the principles of commercial tubes to experimental HPM devices.

One of the most challenging requirements of high power microwave sources is high-current, cold cathodes. Extensive previous research has been performed on such cathodes over the past 30 years. ${ }^{1-7}$ A variety of cathodes were investigated in the past, including:

(1) carbon fiber tufts, ${ }^{1,6-9}$

(2) velvet (carbon, cotton or polyester), ${ }^{4,6}$

(3) carbon felt, ${ }^{10}$

${ }^{\text {a)} E l e c t r o n i c ~ m a i l: ~ r o n g i l g @ u m i c h . e d u ~}$
(4) plasma, ${ }^{5}$ and

(5) bare metal. $^{2}$

The ideal cathode for an HPM device would be one that exhibits the following properties:

(a) high current densities $\left(\mathrm{kA} / \mathrm{cm}^{2}\right)$;

(b) rapid current turn on at moderate electric fields $(\ll 100 \mathrm{kV} / \mathrm{cm})$;

(c) little or no plasma production (after conditioning or cleaning), resulting in no diode impedance collapse over microsecond pulselengths; and

(d) survivability under intense, microsecond current generation, electron and ion backbombardment, as well as postpulse arcs.

Until recently, these ideal cathode properties appeared unattainable. However, two recent cathode developments have shown that these ideal cathode goals may be technically feasible. The first type of cathode is Schiffler's carbon-fiber, cesium iodide-coated cathode. ${ }^{8,9}$ This cathode has been shown to generate long-pulse, high power microwaves in a relativistic magnetron. The Schiffler cathode generates high current densities and survives even under repetitively pulsed operation.

The second, innovative new type of cathode, demonstrated in these preliminary experiments at UM is the Projection Ablation Lithography (PAL) cathode. The PAL cathode utilizes microtexturing of a solid metal cathode substrate to 


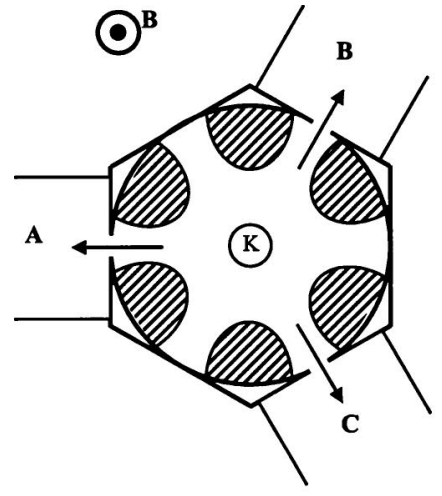

(a)

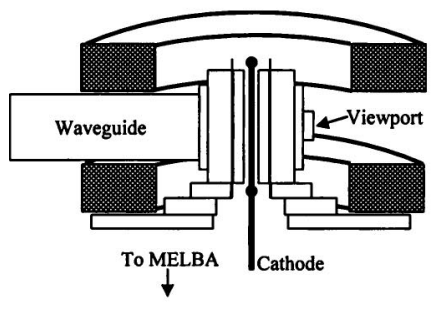

(b)

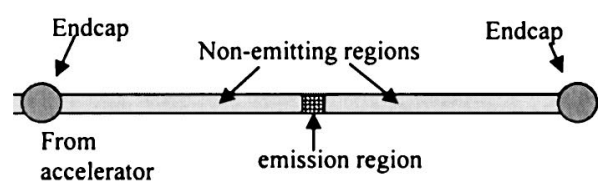

(c)

FIG. 1. Experimental configuration of: (a) the A6 magnetron structure with orientation of the cathode and the three WR-650 waveguides, (b) cross section of the magnetron, cathode, and the magnet coil orientation, and (c) a close up the PAL cathode with the endcaps, emission region, and the nonemitting regions.

provide field enhancement. The advantages of this cathode are that, unlike microtips which can overheat, explode and create plasma, the PAL cathode emission regions can be micromachined with limited electric field enhancement while the emission regions are "heat-sinked" to the base cathode material. Furthermore, the PAL cathode consists of pure metal so after an initial bakeout or plasma cleaning (or a few conditioning shots) there is negligible gas emission (unlike fabric cathodes).

We have demonstrated for the first time that by ablating three azimuthal regions of active current emission on the cathode (Tri-PAL), that the electron beam can be prebunched into three spokes. This process is defined here as "cathode priming" of the pi mode for a six-cavity magnetron.

\section{EXPERIMENTAL CONFIGURATION}

The relativistic magnetron is driven by the Michigan Electron Long Beam Accelerator Ceramic (MELBA-C), with operating parameters: voltage flattop of $-300 \mathrm{kV}$, entrance current of 3-9 kA, and a pulse length of $300 \mathrm{~ns}$. The ceramic insulator results in a base vacuum on the $10^{-8}$ Torr scale, a factor of 100 lower than most HPM devices. Relativistic magnetron experiments at UM have been performed using a six-vane Titan Pulses Sciences tube [Fig. 1(a)]. The Titan tube is based on an unstrapped A6 geometry, with

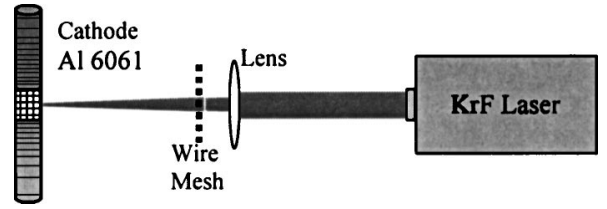

FIG. 2. Experimental configuration used in fabricating the cathodes.

rounded stainless-steel vane tips. The pi mode frequency is about $1.04 \mathrm{GHz}$ and the $2 / 3$ pi mode resonant frequency is 980 MHz. ${ }^{11}$ Three L-Band (WR-650) waveguides are attached to the magnetron for extraction of microwaves as seen in Fig. 1(a). Figure 1(b) shows the cross sectional view of the magnetron with the cathode orientation. Also shown are the two electromagnets, which provide an axial magnetic field; (same value for all shots: $3 \mathrm{kG}$ ). The cathode is shown in Fig. 1(c), with the emission region centered inside the magnetron vanes. Endcaps are located at the end of the cathode and before the magnetron to help prevent electron end-loss current.

Microwaves are extracted out of three of the six cavities in the magnetron, each spaced $120^{\circ}$ from one another; this favors pi-mode operation. The microwaves then travel through Lucite windows into $L$-band waveguide. In one of the waveguides a loop-coupler extracts power and the microwave signal is transmitted to the screen room through RG$214 / \mathrm{U}$ cable. A zero-area $B$-dot loop is located on the waveguide. The remaining two waveguides are terminated in water loads. Microwave signals are heterodyned with a local oscillator at $1.3 \mathrm{GHz}$. This heterodyne signal is sent to a fast oscilloscope and data are processed in a time frequency analysis program to give the frequency of the microwaves as a function of time. ${ }^{12}$ Incoming diode current, endloss electron current, and voltage traces are also measured and sent to the screen room.

Previous cathodes used in the relativistic magnetron at UM were made of an aluminum alloy (Al 6061); after the Al rod was sanded, either carbon or cotton fibers were glued onto the cathode in the middle of the interaction region. Problems with these cathodes included outgassing of the fibers and glue that held them on the cathode, resulting in short microwave pulse lengths. To alleviate problems with the carbon/cotton cathodes, the new technique was employed wherein a pattern was ablated onto the aluminum cathode to provide the field enhancement without the outgassing of cloth.

\section{FABRICATION TECHNIQUE}

To fabricate the cathode, a pattern needed to be projected onto the cathode at sufficiently high laser intensity to ablate the surface. In order to accomplish this, a $600 \mathrm{~mJ} /$ pulse $\mathrm{KrF}$ laser was employed. The cathode stalk (Al 6061 alloy, $1 / 2$ in. diameter) was sanded smooth using 600, 1500, and then 2500 grit sandpaper. After being sanded the cathode rod was cleaned using acetone and methanol. Figure 2 shows the experimental setup of the $\mathrm{KrF}$ laser, focusing lens $(25 \mathrm{~cm}$ focal length), projection mask (wire mesh with $914 \mu \mathrm{m}$ wire diameter with $52 \%$ open area), and the cathode orientation. The ablation laser is a KrF laser (Lambda-Physik Compex 


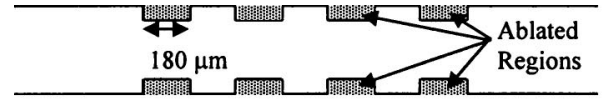

(a)

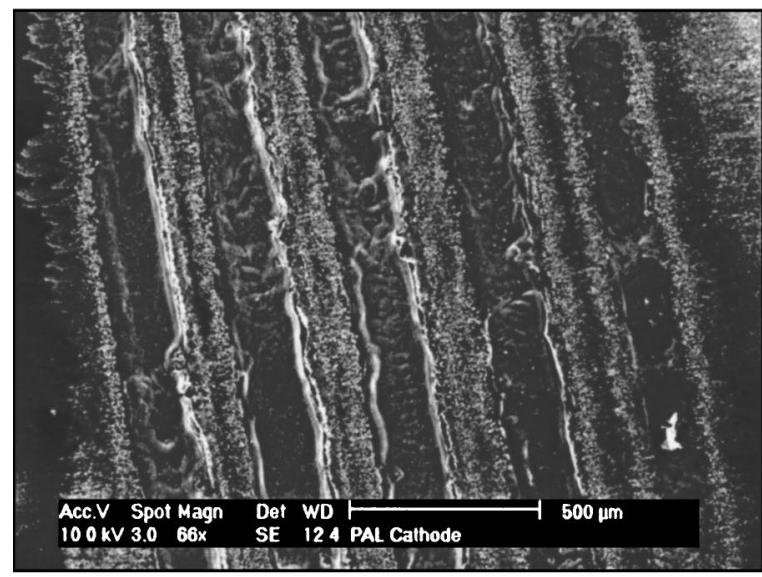

(b)

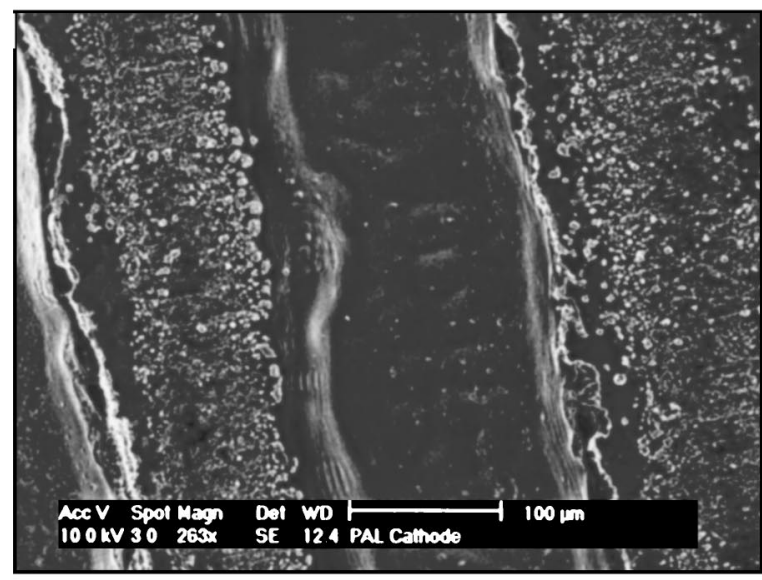

(c)

FIG. 3. Laser ablated pattern on the cathode: (a) shows the cross section where the trench width is $180 \mu \mathrm{m}$ and the depth is approximately $50 \mu \mathrm{m}$. (b) and (c) are SEM images of the ablated pattern which shows the trench created by the laser and the particles which were redeposited on the sample.

205 ) operating at $20 \mathrm{~Hz}, 400 \mathrm{~mJ}, 248 \mathrm{~nm}$ wavelength, and a $20 \mathrm{~ns}$ pulse length. The ablated pattern on the cathode has a size of $9.1 \mathrm{~mm}^{2}$. After the laser was fired for one minute (1200 shots) the $\mathrm{Al}$ cathode rod was rotated by $45^{\circ}$ and the laser was fired for another minute; after rotating $360^{\circ}$ the cathode was shifted $1 \mathrm{~mm}$ along its axis, and the process was repeated. The total area of the pattern on the cathode was initially $4 \mathrm{~cm}^{2}$ (PAL-I) and then changed to $6 \mathrm{~cm}^{2}$ (PAL-II) to increase the emitted current.

A cross-sectional view of the cathode is shown in Fig. 3(a). This view shows the ablated regions on the cathode. Figures 3(b) and 3(c) show scanning electron microscope (SEM) images of the ablated pattern. From Fig. 3(b) the trench width is approximately $180 \mu \mathrm{m}$. The depth of the ablated regions is approximately $50 \mu \mathrm{m}$. Figure 3(c) shows the particles that have been redeposited on the high points of the cathode because the cathodes were processed in atmospheric air. These redeposited particles may contribute to the enhancement of the electric field.

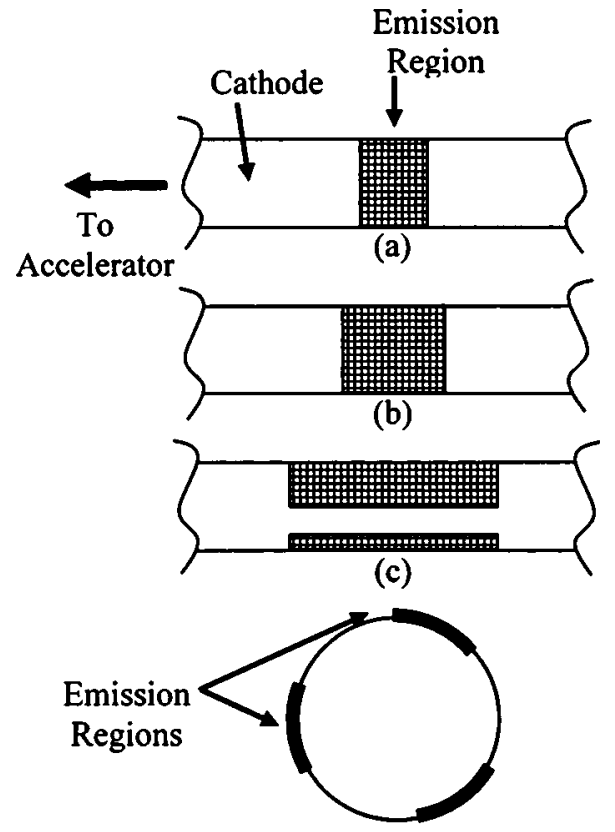

(d)

FIG. 4. Ablated pattern orientation on the three cathodes. (a) and (b) were a solid pattern around the circumference of the rod, with areas of 4 and $6 \mathrm{~cm}^{2}$. (c) Tri-PAL cathode which has three emission regions around the cathode, shown in (d); which is a cross section of the cathode showing the emission regions spaced $120^{\circ}$ apart.

Three different PAL cathodes have been used to date as seen in Fig. 4. PAL-I, shown in Fig. 4(a), had the smallest area of $4 \mathrm{~cm}^{2}$, the axial length was $1 \mathrm{~cm}$. Figure 4(b) shows the PAL-II cathode, in which the area was increased by $50 \%$ to $6 \mathrm{~cm}^{2}$ and the surface was polished smooth with a compound after sanding, but before laser ablation. Tri-PAL, seen in Figs. 4(c) and 4(d), has the same area as PAL-II, $6 \mathrm{~cm}^{2}$, but utilizes three emission regions, spaced $120^{\circ}$ apart to generate the desired three-electron spokes, to help promote faster microwave startup time for the pi mode in the sixcavity magnetron; "cathode priming."

No in situ bakeout was employed in these experiments. Note that the emitting region of the cathode is heated to vaporization by the laser ablation.

\section{EXPERIMENTAL DATA AND ANALYSIS}

The initial projection ablation lithography (PAL-I) cathode tested had an emission area of $4 \mathrm{~cm}^{2}$. The observed electron current was too low for the generation of high-power microwave oscillation; the current averaged $2.8 \mathrm{kA}$. Previous work using a cotton cathode showed that there needed to be between 4 and $6 \mathrm{kA}$ electron beam current to generate highpower microwaves. The power on the first shot of PAL-I was $100 \mathrm{MW}$, however, the average power of the remaining 16 shots was $15 \mathrm{MW}$. The increased current and power on the first shot was probably due to initial release of surface contaminants. Although the source of plasma is believed to be primarily hydrogen, no residual gas analyzer (RGA) was used in the present experiments. Previous UM experiments utilizing an RGA with metal cathodes and a plastic insulator showed that the most abundant contaminant was hydrogen. ${ }^{13}$ 


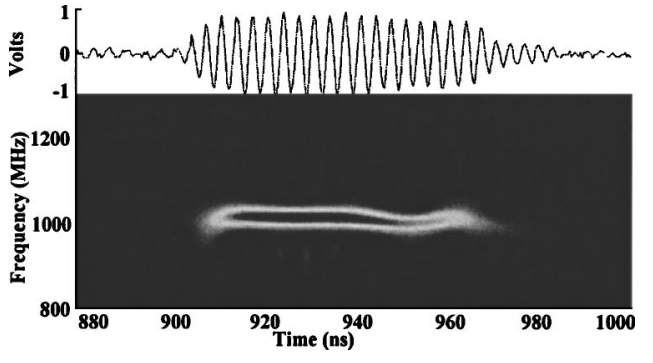

FIG. 5. (Top) Heterodyned microwave signal for PAL-I cathode. (Bottom) Time frequency analysis of heterodyned microwave signal, showing a peak frequency of $1014 \mathrm{MHz}$, which corresponds to the pi mode frequency.

Plasma cleaning experiments at UM showed that this $\mathrm{H}$ contaminant could be reduced or eliminated if the plasma cleaning was operated until within a few seconds of the HPM pulse. $^{2}$ The heterodyned microwave signal for the first shot is shown in the upper half of Fig. 5. This heterodyned signal, after being processed in the time frequency program, shows the frequency versus time, shown in the lower half of Fig. 5. The frequency for this shot was $1.014 \mathrm{GHz}$, which is the pi mode frequency.

To increase the electron current, PAL-II cathode was fabricated having a larger emission area, increased from 4 to $6 \mathrm{~cm}^{2}$. The new average current was $3.7 \mathrm{kA}$. This increase in current led to the microwave power increasing by a factor of 2.5, from 15 to $40 \mathrm{MW}$. Figure 6(a) shows the entrance current and voltage for a typical PAL-II cathode shot; the voltage has a flattop around $300 \mathrm{kV}$, and the current ramps from 3 to $6 \mathrm{kA}$ on the voltage flattop. The average microwave startup time was $126 \mathrm{~ns}$, with a standard deviation of $22 \mathrm{~ns}$. The microwave startup is defined here as the time microwave oscillation starts relative to the time at $10 \%$

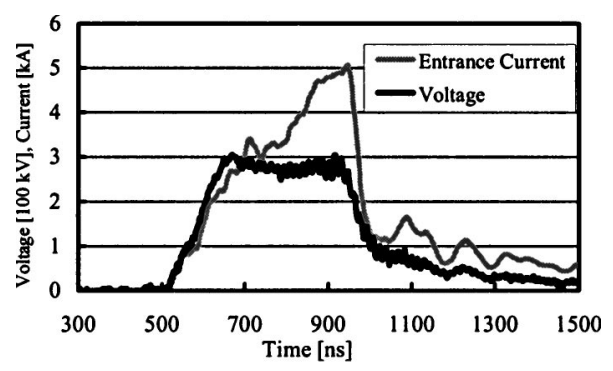

(a)

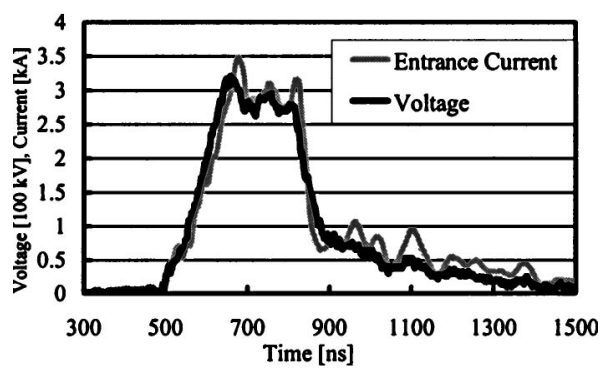

(b)

FIG. 6. Voltage and current traces for cathodes with: (a) single emission region and (b) triple emission regions (Tri-PAL). of the voltage pulse maximum amplitude. The average pulse length was the longest for the PAL-II cathode with the microwave oscillation lasting $288 \mathrm{~ns}$.

The Tri-PAL cathode was made with three emission regions to promote faster startup (cathode priming) by prebunching the electrons into three spokes to generate the pi mode inside the six-cavity magnetron. ${ }^{14,15}$ The average microwave startup time decreased to $110 \mathrm{~ns}$ with the Tri-PAL cathode and the standard deviation of the startup was reduced to $9.6 \mathrm{~ns}$. This is nearly the fastest microwave startup time possible with this voltage pulse, since the voltage rise time is now on the order of the microwave startup time. Another important property of the Tri-PAL cathode is that in approximately $40 \%$ of the shots, the current followed the voltage with no measurable plasma closure, as seen in Fig. 6(b).

Closure velocities of the plasma were calculated for the three PAL cathodes for comparison to shots of the previous cotton cathodes. The closure velocity is found by plotting $1 / \sqrt{P}$, where $P$ is the perveance, and comparing this to the measured values. The perveance is found from Eq. (1) and is derived from Langmuir ${ }^{16}$ and Dow. ${ }^{17}$ This equation is simply the Child-Langmuir law in one dimension (1D) for currents limited by space charge between coaxial cylinders

$$
I \approx 14.68 \times 10^{-6} \frac{L V^{3 / 2}}{r_{\rho} \beta^{2}},
$$

where $L$ is the effective length of the emission region, $r_{p}$ is the anode radius, and $\beta$ is a function of the anode radius and the cathode radius which varies with time and the closure velocity.

Figure 7 plots $1 / \sqrt{P}$ for the two shots shown in Fig. 6 . Figure 7(a) shows the case for the single-emission region cathode in which plasma closure existed, with a velocity of $2.35 \mathrm{~cm} / \mu \mathrm{s}$. Figure 7(b) shows the Tri-PAL cathode case when the current followed the voltage trace; as seen in Fig. $6(\mathrm{~b})$, the closure velocity here was found to be $0.5 \mathrm{~cm} / \mu \mathrm{s}$. The average closure velocity of the cotton cathode was $2.7 \mathrm{~cm} / \mu \mathrm{s}$ compared to PAL-I closure velocity of $2.1 \mathrm{~cm} / \mu \mathrm{s}$, and PAL-II closure velocity of $2.9 \mathrm{~cm} / \mu \mathrm{s}$. A crucial finding for the Tri-PAL cathode is that there were two distinct regions for the closure velocity. Region one occurred when plasma closure existed, which led to an average velocity of $1.9 \mathrm{~cm} / \mu \mathrm{s}$; whereas in the second region the closure was extremely low, averaging $0.2 \mathrm{~cm} / \mu \mathrm{s}$, with some shots showing zero plasma closure (shots in which the slope of $1 / \sqrt{P}$ is positive).

\section{DISCUSSION}

Figure 8 compares the performance of two types of PAL cathodes, single, and triple emission regions, as well as the cotton fiber cathode. (Data are not compared to previous carbon fiber cathode; since that cathode was utilized in a twowaveguide extraction and the table only compares threewaveguide extraction.) From the figure it is shown that the microwave startup time and microwave pulselength improve dramatically with PAL cathodes, while the single-waveguide power levels have roughly remained constant. Power mea- 


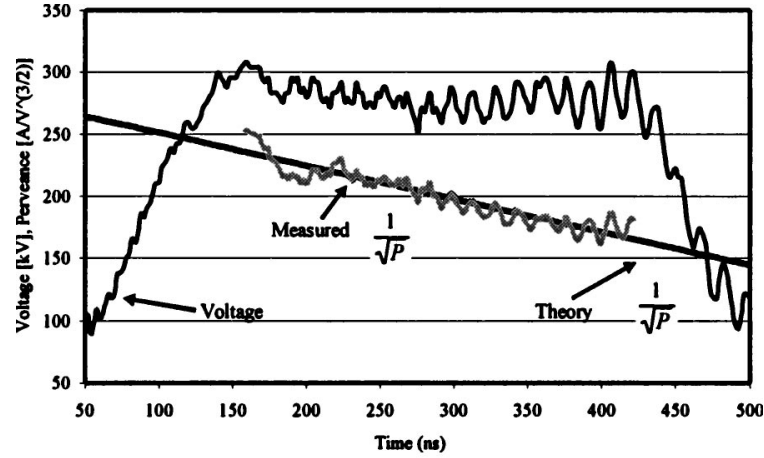

(a)

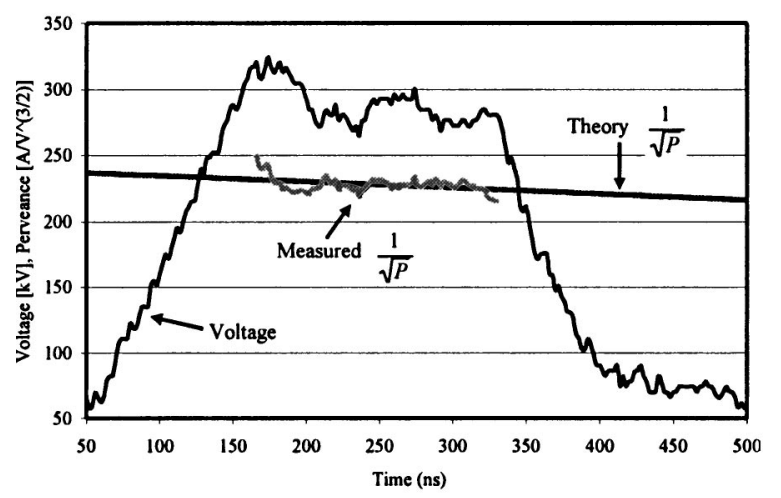

(b)

FIG. 7. Plots of $1 / \sqrt{P}$ of the same shots as in Fig. 6, where $P$ represents the perveance. (a) Single emission case when there is plasma closure, with a velocity of $2.35 \mathrm{~cm} / \mu \mathrm{s}$. (b) Triple emission case (Tri-PAL) when the plasma closure velocity is reduced to $0.5 \mathrm{~cm} / \mu \mathrm{s}$, e.g., when the current nearly follows the voltage.

surements for the single emission PAL cathodes are not shown in Fig. 8. The microwave power signals were extremely noisy and therefore could not be compared directly with other cathodes using the same $60 \mathrm{MHz}$ low-pass filter. Peak microwave electronic efficiency $\left(P_{\text {microwaves }} / I V\right)$ was $13 \%$ for the cotton cathode, $9 \%$ for the single emission PAL cathode, and was increased to $17 \%$ for the Tri-PAL cathode. The efficiency calculations assume that all three waveguides

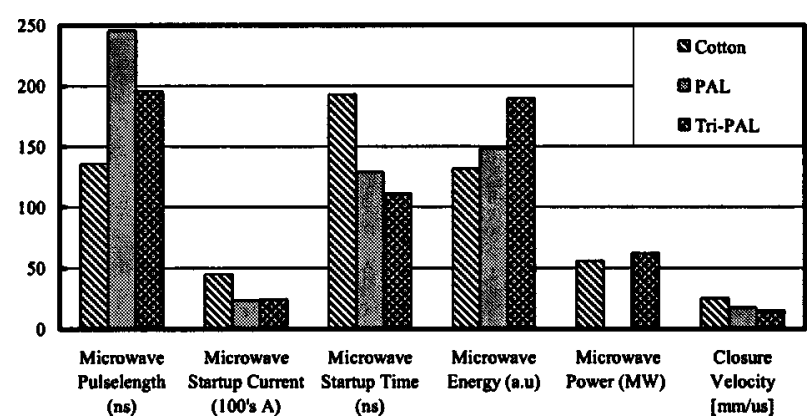

FIG. 8. Comparison of the PAL cathode, single emission region, and triple emission region (Tri-PAL) with the previous cotton fiber cathode. Microwave power measurements are from a single-waveguide; total extracted microwave power is expected to be a factor of 3 higher than shown. are extracting the same microwave power, and therefore the microwave power signals is multiplied by three.

In conclusion, a projection ablation lithography (PAL) high-current cathode has been developed and demonstrated to generate high-power microwaves in a relativistic magnetron. This cathode demonstrates rapid-current turn-on, low vacuum base-pressure and no significant degradation over 10 's of shots. A new triple-emission region (Tri-PAL) cathode has been shown to provide a technique for "cathode priming"of a magnetron to prebunch the electrons into the desired three spokes for the pi mode in a six-cavity magnetron. The microwave starting time for the Tri-PAL cathode is the lowest of the cathodes tested and the electronic efficiency is the highest.

Future research will focus on the fundamental electron emission mechanisms of the PAL cathode and the physics of shots that exhibit zero closure and correlation to the plasma. Future experiments will utilize an RGA to further study gas emission from PAL cathodes. Also the angular orientation of the Tri-PAL cathode represents an important future area of interest. Processing of PAL cathodes in vacuum will reduce the redeposited particles.

\section{ACKNOWLEDGMENTS}

This research has been supported by the Air Force Office of Scientific Research and by the Air Force Research Lab (AFRL). The authors also acknowledge equipment loans from AFRL.

${ }^{1}$ R. M. Gilgenbach et al., Proceedings of the 5th IEEE Pulsed Power Conference, Arlington, VA, June 10-12 1985, p. 126, IEEE Catalog number $85 \mathrm{C} 2121-2$.

${ }^{2}$ J. I. Rintamaki, R. M. Gilgenbach, W. E. Cohen, R. L. Jaynes, M. E. Cuneo, and P. R. Menge, Appl. Phys. Lett. 75, 31 (1999).

${ }^{3}$ M. R. Lopez et al., IEEE Trans. Plasma Sci. 30, 947 (2002).

${ }^{4}$ R. B. Miller, J. Appl. Phys. 84, 3880 (1998).

${ }^{5}$ D. M. Goebel, J. M. Butler, R. W. Schuacher, J. Santoru, and R. L. Eisenhart, IEEE Trans. Plasma Sci. 22, 547 (1994).

${ }^{6}$ D. Shiffler, M. Ruebush, M. LaCour, K. Golby, R. Umstattd, M. C. Clark, J. Luginsland, D. Zagar, and M. Sena, Appl. Phys. Lett. 79, 2871 (2001).

${ }^{7}$ D. Shiffler, M. Ruebush, M. Haworth, R. Umstattd, M. LaCour, K. Golby, D. Zagar, and T. Knowles, Rev. Sci. Instrum. 73, 4358 (2002).

${ }^{8}$ D. A. Shiffler, M. J. LaCour, M. D. Sena, M. D. Mitchell, M. D. Haworth, K. J. Hendricks, and T. A. Spencer, IEEE Trans. Plasma Sci. 28, 517 (2000).

${ }^{9}$ D. A. Shiffler, M. J. LaCour, K. Golby, M. D. Sena, M. D. Mitchell, M. D. Haworth, K. J. Hendricks, and T. A. Spencer, IEEE Trans. Plasma Sci. 29, 445 (2001).

${ }^{10}$ R. A. Bosch, H. Ching, R. M. Gilgenbach, P. L. G. Ventzek, P. R. Menge, J. J. Choi, and T. A. Spencer, Rev. Sci. Instrum. 62, 1779 (1991).

${ }^{11}$ D. Price and J. N. Benford, IEEE Trans. Plasma Sci. 26, 256 (1998).

${ }^{12}$ C. W. Peters, R. L. Jaynes, Y. Y. Lau, R. M. Gilgenbach, W. J. Williams, J. M. Hochman, W. E. Cohen, J. I. Rintamaki, D. E. Vollers, and T. A. Spencer, Phys. Rev. E 58, 6880 (1998).

${ }^{13}$ J. I. Rintamaki, Ph.D. thesis, University of Michigan, 1999.

${ }^{14}$ V. B. Neculaes (private communication 2003).

${ }^{15}$ M. C. Jones, R. M. Gilgenbach, W. M. White, M. R. Lopez, V. B. Neculaes, Y. Y. Lau, T. A. Spencer, and D. Price, Proceedings of 31st International Conference on Plasma Science, Baltimore, MD, June 28-July 1, 2004

${ }^{16}$ I. Langmuir and K. B. Blodgett, Phys. Rev. 22, 347 (1922).

${ }^{17}$ W. G. Dow, Fundamentals of Engineering Electronics (Wiley, New York, 1952). 